\title{
Anatomical Variation of the Obturator Artery: A Case Report
}

\author{
Nathalie de Almeida Silva, ${ }^{1}$ Ana Beatriz Marques Barbosa, ${ }^{2}$ Priscele Viana dos Santos, ${ }^{2}$ Vanessa Apolonio Targino, ${ }^{2}$ \\ Felippe Barbosa Gomes, ${ }^{3}$ Yanka Costa de Melo Silva ${ }^{1}$ Thiago de Oliveira Assis ${ }^{3,4,5}$
}

${ }^{1}$ Academics of the Medicine course, University Center of UNIFACISA, Campina Grande, PB, Brazil

${ }^{2}$ Academics of the Physiotherapy Course, University Center of UNIFACISA, Campina Grande, PB, Brazil

${ }^{3}$ Department of Medicine and Physiotherapy, University Center of UNIFACISA, Campina Grande, PB, Brazil

${ }^{4}$ Department of Biology, State University of Paraíba, Campina Grande, Pb, Brazil

${ }^{5}$ Academic Unit of Medicine, Federal University of Campina Grande, Campina Grande, PB, Brazil

Disclose and conflicts of interest: none to be declared by all authors

\begin{abstract}
Introduction: introduction The obturator artery $(\mathrm{OA})$ usually arises from the anterior trunk of the internal iliac artery. In the pelvis, it presents an anteroinferior course to the lateral wall, leading to the medial portion of the thigh, where it is divided into anterior and posterior branches. Through these, it provides supply to the pelvis and lower limbs structures. Although in most cases the OA follows the normal anatomical pattern, this is one of the structures with the greatest variations described. Objective: to describe morphometric aspects of a unilateral anatomical variation of the obturator artery originating from the posterior trunk of the internal iliac artery.

Method: this is a case report, in which the anatomical variation was observed during a routine class in the anatomy laboratory at a university of Paraíba, Brazil, in 2018. A digital caliper was used to register morphometric aspects of the variant OA. Case report: when evaluating an adult male cadaver, a unilateral variation of the left OA was observed, which originated from the posterior division of the internal iliac artery. In the pelvis, the left OA presented an inferolateral course to the common trunk of the inferior gluteal and internal pudendal arteries. The right OA followed the anatomic-normal pattern. Conclusion An origin and course variability of the OA may have important implications in pelvic surgeries. Inadvertent lesions of this artery can lead to profuse and sometimes lethal bleeding. Therefore, it is important that surgeons and radiologists are aware of the possible variations of this structure for an adequate management of this region.
\end{abstract}

Keywords: Pelvis; Obturator artery; Anatomical variation; Vascularization.

\section{Introduction}

The obturator artery (OA) commonly originates from the anterior trunk of the internal iliac artery, following an anteroinferior course over the lateral wall of the pelvis, being crossed in its medial face by the ureter and, in men, by the vas deferens. In the pelvis, it provides iliac branches to the iliac bone and muscle, and also a bladder branch to the bladder. It is directed to the medial portion of the thigh through the obturator canal (foramen between the obturator membrane and the internal obturator muscle), accompanied by the vein (inferior) and obturator (superior), dividing into anterior and posterior branches to supply the muscles from the medial compartment of the thigh; and the femur head and muscles inserted in the ischial tuberosity, respectively. ${ }^{1}$

The OA is one of the anatomical structures with the greatest variation described. In addition to the normal course, its origin has been documented from the common iliac artery, external iliac, inferior epigastric, internal pudgy, lower gluteal, gluteal, iliolumbar, and as direct branches of the posterior trunk of the internal iliac artery. ${ }^{2}$ It may present double or even triple origin, ${ }^{2}$ or be absent, being replaced by the deep posterior trunk of the femoral artery. ${ }^{3}$

Anatomical studies have commonly observed that, in most cases, the origin of the OA follows the normal anatomical pattern, from the anterior trunk of the internal iliac artery. In India, a study with 72 hemipelves, from both genders, observed that the $\mathrm{OA}$ origin through the internal iliac artery occurred in $93 \%$ of the cases, being $69 \%$ from the anterior division. ${ }^{4}$ Similar data were observed in the United Kingdom (50.9\%)2 and in Brazil (59.1\%). ${ }^{5}$ The origin of this artery as a direct branch of the posterior trunk of the internal iliac artery is one of the most unusual variations, being observed in $\sim 0.5 \%$ to $12.5 \%$ of the cases described. ${ }^{4-9}$ Data from a meta-analysis performed from a cadaveric study from more than 3,000 hemipelves, of both genders, observed a prevalence of $12.1 \%$ of this anatomical variation. ${ }^{2}$

This variability in the OA origin has attracted the attention of surgeons due to its importance as 
a source of potentially lethal bleeding in urologic, gynecological and for inguinal and femoral hernia repairs, ${ }^{2}$ as well as orthopedic surgeries (see Fig. 1). The results from the present study were similar to those reported in the literature. Thus, surgeons need to be aware of unexpected sources of bleeding, taking into account variations in the retropubic vascular anatomy, so that they can take appropriate precautions to avoid inadvertent injury of these structures. ${ }^{4}$
Inaddition, knowledgeabout the possibleanatomical variations involving this region is important for an adequate interpretation of angiographic studies of the pelvic vessels. ${ }^{6}$ The objective of the present study was to describe the morphometric aspects of a unilateral anatomical variation of the obturator artery originating from the posterior trunk of the internal iliac artery.
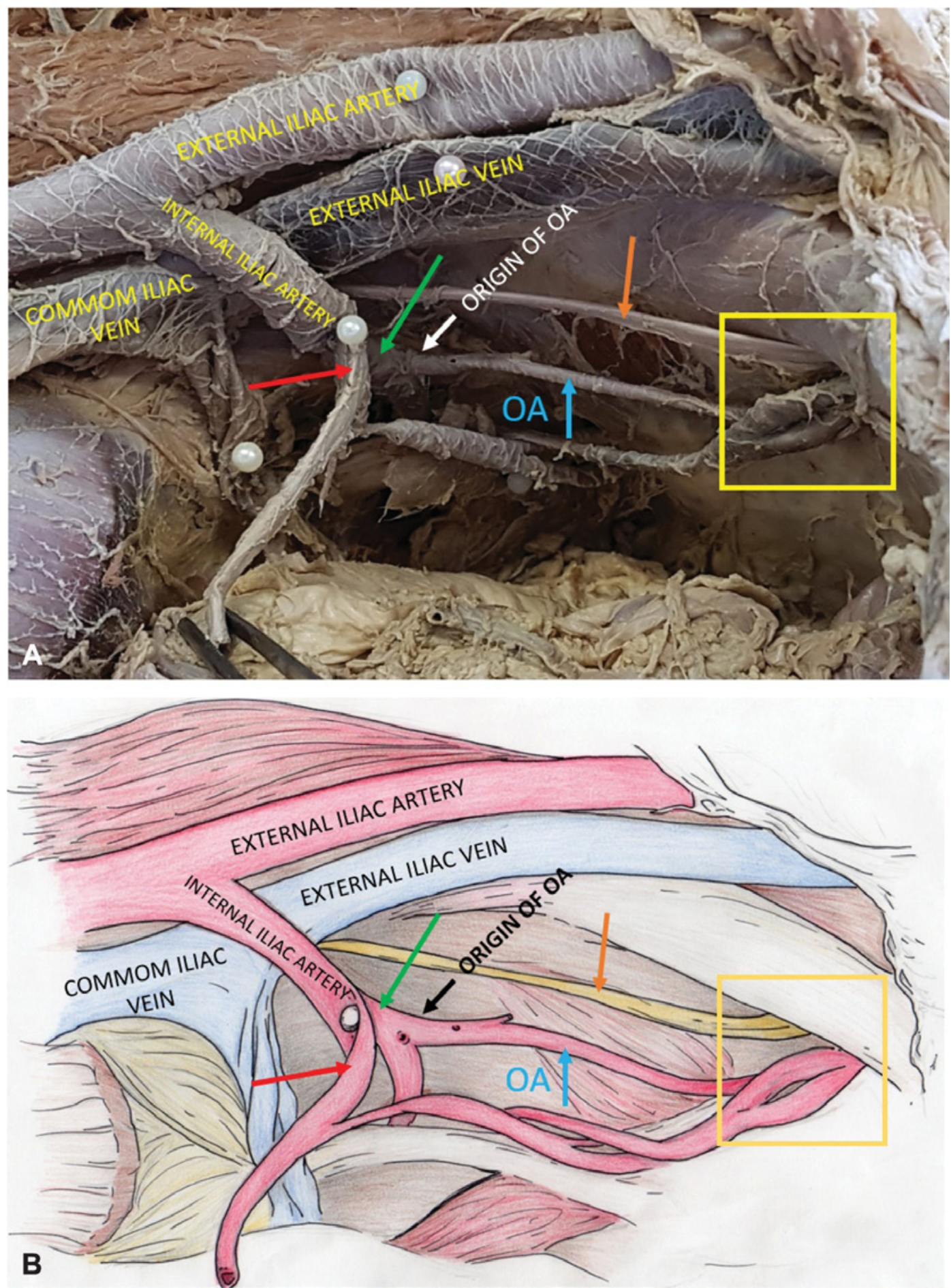

Figure 1. Vessels and nerves passing through the upper opening of the true pelvis, corpse photography (A), and schematic figure (B). Notice the obturator artery (OA) origin (blue arrow) originating from the posterior trunk of the common iliac artery toward the obturator canal (yellow square). Adjacent structures: anterior (red arrow) and posterior (green arrow) trunk of the internal iliac artery, obturator nerve (orange arrow). 


\section{Materials and Method}

This is a case report about an anatomical variation observed in the left hemiple of an adult male corpse from a dissection in the anatomy laboratory at a university in Paraíba, Brazil. The following characteristics of the variant OA were described: vessel from which the artery originated, distance from its origin to its exit in the obturator foramen, pelvic course, proximal diameter (PD), distal diameter (DD) and distance from the origin of the anterior (DRA) and posterior (DRP) branches from the pelvis OA exit (Fig. 1). For the morphometric evaluation a digital caliper was used (Stain-lesscaliper 6 ' 150 mm, Steinless Hardened, Pinhais, Paraná, Brazil). The anatomical specimen was photographed for confirmation and characterization of the studied artery.

\section{Case Report}

During an anatomy class, an anomalous origin of the left OAwas observed from the posterior division of the internal iliac artery from an adult male corpse. The artery presented an infero-lateral course to the common trunk of the inferior gluteal and internal pudendal arteries, passing under the obturator nerve, over the obturator vein and medial to the internal obturator muscle and its fascia. The left pelvis OA length (distance from the point of origin to the obturator foramen) was $82.49 \mathrm{~mm}$.

The OA exited the pelvis through the upper portion of the obturator canal, toward the medial compartment of the thigh. It bifurcated into anterior and posterior branches at the thigh level. The anterior branch originated $4.35 \mathrm{~mm}$ and the posterior branch $8.33 \mathrm{~mm}$ after its exit through the obturator canal. There was a discrete difference between the PD $(19.54 \mathrm{~mm})$ and the DD $(17.91 \mathrm{~mm})$ of the left PD. The right PD followed the common anatomic pattern of this artery, arising from the anterior division of the internal iliac artery to the obturator canal.

\section{Discussion}

Anatomical variations in the origin and course of the major pelvic arteries have long attracted the attention of anatomists, radiologists and surgeons, especially those involved in procedures in the retroinguinal (Retzius) and in the retro-pubic (Bogros) space..$^{10}$ Vessels injuries of this region tend to bleed profusely, with considerable technical difficulty to bind them. ${ }^{11}$ One of the main bleeding sources is related to the anomalous origin of the OA. ${ }^{2}$ Although, in most cases, the OA originates from the anterior division of the internal iliac artery, ${ }^{3}$ the incidence of anatomical variations of this artery varies from 13 to $30 \% .^{12}$ Arterial bleeding is a serious and potentially lethal complication in pelvic injuries involving branches of the internal iliac artery. ${ }^{4}$ The origin of the $\mathrm{OA}$ as a direct branch from the posterior division of the internal iliac is one of the most unusual variations, documented more frequently in the Indian population. .,9,10,12 $^{2}$

In a study conducted in New Delhi, India, among 316 hemipelves dissected, only 1 case $(0.5 \%)$ of this variation was observed. ${ }^{6}$ Another Indian study observed this variation in $11.7 \%$ of anatomical specimens, the highest occurrence in male pelvis (71.4\%; 5 cases) when compared with female $(28.6 \%$; 2 cases). ${ }^{8}$ A study conducted in the United Kingdom observed a prevalence of $8.2 \%$ of this variation, and unlike the study by Sakthivel et al, it occurred more frequently in females (15 cases) than males (4 cases). ${ }^{2}$ In Brazil, a study evaluating 56 hemipelves, from both genders, found a variation in the OA origin in 33.9\% of the dissected parts; however, in none of them did the OA originate from the posterior division of the internal iliac artery, ${ }^{13}$ unlike Tajra et al. ${ }^{11}$ who verified this variation in $18.2 \%$ of the hemipelves evaluated. In the latter, all cases presented an OA course through the retroinguinal space, on the superior branch of the pubis.

The proximity of obturator vessels to the superior branch of the pubis may result in persistent hemorrhage associated with inadvertent injuries during ilioinguinal incision (retro-pubic fat may make it difficult to see the vessel), or in orthopedic surgeries involving the fixation of acetabular pelvic ring fractures (thus, the posterior superior-superior area of the pubis should be carefully palpated to detect pulsatile activity. ${ }^{14}$ In addition, the proximity of the OA to the lacunar ligament also makes the vessel vulnerable during ligament release for open or endoscopic hernioplasty of femoral canal hernias. ${ }^{15}$ Herniorrhaphy for reconstruction of the inguinal canal floor requires access through the Bogros space, which may also expose injuries in this vessel. ${ }^{9}$

Variations in the OA origin appear to be related to unusual embryologic selections of channels of a primary capillary plexus, in which some develop while the others retract. There-fore, it is probable that there is a development of a primitive OA due to the posterior division of the internal iliac artery, because of a delay in the development of the channels in the primitive anterior division. Thus, these tend to disappear by establishing the final arterial pattern from the posterior trunk. ${ }^{16}$

An advantage attributed specifically to this anatomical variation is related to the formation of collaterals to the femoral head in cases of ischemic necrosis due to aortoiliac and femoral artery occlusive diseases. In this case, a possible bypass graft can be considered by connecting the posterior division to the distal end of the obstruction. 6 In addition, in procedures for ligation of the internal iliac arteries and their branches in women undergoing pelvic surgery, such as hysterectomy, the OA will be spared, ${ }^{10,16}$ reducing possible complications in vascular supply to the lower limbs. 


\section{Conclusion}

Obturator artery variations are not uncommon findings, with different anatomic patterns being reported, among them, their origin from the posterior trunk of the internal iliac artery. Thus, knowledge about retropubic vascular anatomy as well as the possible variations that may occur in this region are important for surgical planning to minimize bleeding or prevent subsequent acute or late ischemia; as well as adequate imaging evaluation tests related to the pelvic and inguinal regions.

\section{References}

1. Standring S. Gray's Anatomy the anatomical basis of clinical practice. 40th ed. London: ElsevierChurchilLivingstone; 2010.

2. Sañudo JR, Mirapeix R, Rodriguez-Niedenführ M, Maranillo E, Parkin IG, Vázquez T. Obturator artery revisited. Int Urogynecol J Pelvic Floor Dysfunct 2011;22(10):1313-1318.

3. Bergman RA, Thompson SA, Afifi AK, Saadeh FA. Compendium of human anatomic variation. Baltimore: Urban\&Schwarzenberg; 1988 4. Suryavanshi A, Bhagel P, Sharma D. Variability in the origin of the obturator artery: A descriptive cross-sectional cadaveric study. IJSS Journal of Surgery. 2016;2(02):16-19.

5. Pick JW, Barry J, Anson BJ, Ashley FL. The origin of obturator artery - a study of 640 body halves. Am J Anat 1942;70:317-344.

6. Kumar D, Rath G. Anomalous origin of obturator artery from the internal iliac artery. Int J Morphol 2007;25(03):639-641.

7. Jusoh AR, Abd Rahman N, Abd Latiff A, et al. The anomalous origin and branches of the obturator artery with its clinical implications. Rom J Morphol Embryol 2010;51(01):163-166.

8. Sakthivel PS. Variability of origin of obturator artery and its clinical significance. Int J Anat Res. 2015;3(04):1704-1709.

9. Rajive AV, Pillay M. A study of variations in the origin of obturator artery and its clinical significance. J Clin Diagn Res 2015;9(08):

\section{AC12-AC15.}

10. Pai MM, Krishnamurthy A, Prabhu LV, Pai MV, Kumar SA, Hadimani GA. Variability in the origin of the obturator artery. Clinics (São Paulo) 2009;64(09):897-901.

11. Tajra JBM, Lima CF, Pires FR, Sales L, Junqueira D, Mauro E. Variability of the obturator artery with its surgical implications. J Morphol Sci. 2016;33(02):96-98.

12. Kumari S, Gowda MST. A study of variations of origin of obturator artery: Review in south Indian population. J Anat Soc India 2016; 65(01):S1-S4.

13. Heins FF, Silva JML, Nakashima PSP, et al. Artéria obturatória: Variabilidade anatômica e morfometria de sua origem à bifurca- ção da artéria ilíaca comum. Revista de Saúde. 2011;2(02):13-18.

14. Letournel $E$. The treatment of acetabular fractures through the ilioinguinal approach. Clin Orthop Relat Res 1993;(292):62-76.

15. Boutefnouchet T, Bassett J, Patil S. Anatomy and clinical relevane of the 'corona mortis': A reviewof the literature and current aspects of management. J Orthop Rheumatol 2016;3(02):5.

16. Al-Talalwah W, Al-Hashim Z, Al Dorzi S, et al. The clinical significance of the obturator artery in origin variability. Indian J Soc Res 2016;7(01):61-65.
Received: October 5, 2019

Accepted: October 16, 2019
Corresponding author

Thiago de Oliveira Assis

Email: thiago.oa@hotmail.com 